\title{
ESTIMACIÓN DEL VOLUMEN DE AGUA DE ORIGEN SUBTERRÁNEO PARA UN HUMEDAL COSTERO
}

\author{
Enrique A. SÁNCHEZ CAMACHO* y Manuel MARTÍNEZ MORALES
}

Instituto Mexicano de Tecnología del Agua. Paseo Cuauhnáhuac 8532, Progreso, Jiutepec, Morelos, México, C. P. 62550

*Autor para correspondencia: esanchez@tlaloc.imta.mx

(Recibido junio 2015; aceptado marzo 2016)

Palabras clave: balance hídrico, caudal ecológico, humedales

\section{RESUMEN}

Una de las actividades relevantes para la gestión de recursos hídricos en los últimos años es la consideración del ambiente como un actor principal dentro de la cuenca. Para transitar de la teoría a la práctica en este tema, es necesario establecer un diagnóstico en términos de volumen de agua. Es decir, asociar las condiciones que guarda el ambiente con el volumen de agua existente en la cuenca, además de la que ésta recibe. A partir de tal vinculación es factible establecer un diagnóstico. Este trabajo muestra el caso de la zona conocida como Soconusco en la costa del Estado de Chiapas en el sur de México, lugar donde existen cuatro ríos principales (Cintalapa, Despoblado, Vado Ancho y Huixtla), que drenan la mayor parte del agua que escurre en la cuenca, en primera instancia hacia la reserva de la biósfera (RB) La Encrucijada y posteriormente hacia el Océano Pacífico en interacción con el sistema lagunar que ahí existe. Se realizó un balance del agua subterránea, en el que se estimó el volumen que descarga el acuífero Acapetahua hacia el mar. Posteriormente, también se estimó el volumen escurrido por medio de la precipitación y el resultado se contrastó con el registro hidrométrico existente. En el periodo de estiaje la diferencia entre los dos últimos valores proporciona el volumen que aporta la fuente subterránea, su valor medio anual es aceptablemente cercano (20\% de diferencia) al volumen de drenaje natural obtenido del balance de agua subterránea. Por lo anterior se asume que es el valor mínimo que escurre hacia la RB La Encrucijada (promedio por año) con la información empleada.

Key words: water balance, environmental flows, wetlands

\begin{abstract}
One of the outstanding activities in the water resources management in recent years is the consideration of the environment as a major player within the watershed. To move from theory to practice in this topic, it is necessary to establish a diagnosis in terms of water volume. In other words, to associate each condition that sustains the environment, mainly the inflow of water into the basin. This work shows the case of the zone known as Soconusco in the cost of Chiapas state, in south Mexico, where there are four main rivers: Cintalapa, Despoblado, Vado Ancho and Huixtla. Those rivers drain most of the running off in the basin, first to the biosphere reserve (BR) La Encrucijada and then to the Pacific Ocean, interacting with the lagoon system there. A groundwater balance
\end{abstract}


was done, estimating the volume that the aquifer Acapetahua discharges into the sea. Subsequently, the draining volume was estimated by precipitation and the result was checked with the hydrometric record. In the dry season period the difference between the last two values provides the volume of water that comes from the underground source. Its annual average value is acceptably close (20\% difference) to the volume of natural drainage obtained from the groundwater balance. Therefore, it was assumed that the minimum value drains into La Encrucijada BR (average per year) with the available information.

\section{INTRODUCCIÓN}

En junio de 2011 se publicó el documento "Identificación de reservas potenciales de agua para el medio ambiente en México" (CONAGUA 2011), producto del trabajo de la alianza del Fondo Mundial para la Naturaleza (WWF, por sus siglas en inglés) y la Fundación Gonzalo Río Arronte en conjunto con un grupo de más de 100 especialistas. Estos especialistas provenientes de 27 instituciones académicas y organizaciones, entidades de gobierno, usuarios de agua y comunidades. El documento fue auspiciado por la Comisión Nacional del Agua (CONAGUA), su objetivo era la determinación de un caudal ecológico para tres ríos en el mismo número de cuencas.

Para 2012, ambas fundaciones con la CONAGUA, el financiamiento del Banco Interamericano de Desarrollo y el apoyo de la Comisión de Áreas Naturales Protegidas, iniciaron el Programa Nacional de Reservas de Agua, con el propósito de reservar por decreto agua para el ambiente (Salinas 2015). Asimismo, actualmente existe el sitio "Reservas potenciales de agua para el medio ambiente" que funciona a través de un mapa interactivo con varias capas de información (CONAGUA 2014).

Como coronación de todos estos trabajos en septiembre de 2012 se publicó la Norma Mexicana NMX-AA-159-SCFI-2012, que establece el procedimiento para la determinación del caudal ecológico en cuencas hidrológicas (SE 2012). Esta norma generó un buen número de trabajos en el país, baste citar que en las Memorias del V Congreso Mexicano de Ecología existen al menos cuatro trabajos que se dedican explícitamente al tópico.

Esta pequeña reseña indica que existe un marcado interés en establecer la cantidad de agua que debe tener el ambiente, porque es alrededor de esta idea que está escrita la norma antes referida, aunque se presenten en su contenido diferentes métodos para establecer el caudal ecológico, algunos con más o con menos variables.
A diferencia de estos trabajos, presentamos un análisis a partir de los registros históricos que existen, se han omitido las mediciones realizadas en campo por espacio y para evitar dispersión en el tema, y su proceso a través de los métodos aceptados en la hidrología de superficie y la geohidrología.

\section{MATERIALES Y MÉTODOS}

\section{Sitio de estudio}

La zona de estudio pertenece a la región hidrológica 23 de la costa del estado de Chiapas, en específico se trabajó con la denominada subregión Huixtla. El área de interés abarca tres subcuencas, cuyos nombres oficiales son: 1) Laguna del Viejo y Tembladeras, con una extensión superficial de $892.56 \mathrm{~km}^{2}$, 2) río Despoblado, con una superficie de $605.11 \mathrm{~km}^{2}$ y 3) río Huixtla, con una extensión de $828.97 \mathrm{~km}^{2}$, (INEGI 2011). En total el área de estudio tiene una superficie de $2326.64 \mathrm{~km}^{2}$ (Fig. 1).

En las tres subcuencas existe una densa red hidrográfica, en la que sobresalen cuatro ríos por los que escurre la mayor parte del volumen que precipita en la zona y el caudal base: río Cintalapa, río Vado Ancho, río Despoblado y río Huixtla (Fig. 2). Los ríos Cintalapa y Vado Ancho se encuentran en la subcuenca Laguna del Viejo y Tembladeras, el río Despoblado y el río Hiuxtla en las subcuencas que llevan el mismo nombre, respectivamente. En la misma figura se muestra la ubicación geográfica de las cinco estaciones hidrométricas que existen en la zona de estudio (SEMARNAT, CONAGUA, IMTA 2008). Es necesario señalar que la estación hidrométrica denominada Compoapa está ubicada en el cauce de uno de los tributarios del río Despoblado. Para este trabajo se seleccionaron las estaciones climatológicas convencionales (IMTA 2010), cuya posición relativa y sus correspondientes polígonos de Thiessen se muestran por subcuenca en la figura 3.

En cuanto al agua subterránea tenemos que el principal acuífero de la zona de estudio es el 


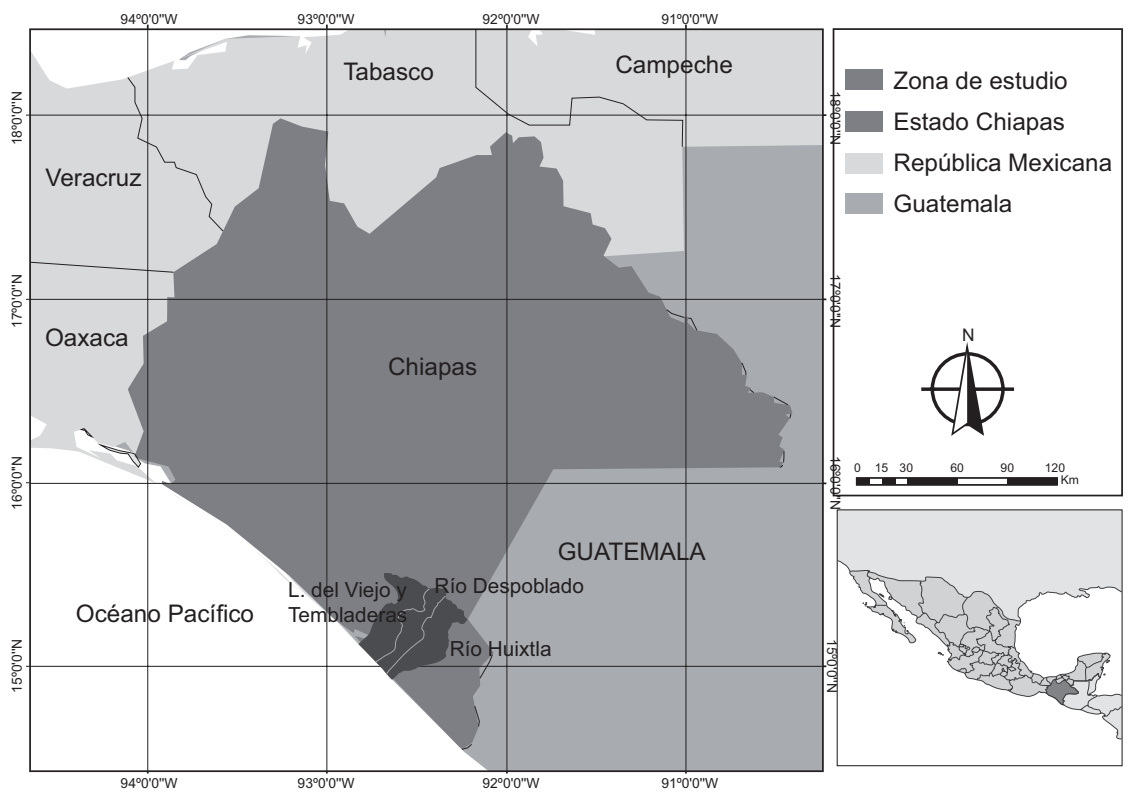

Fig. 1. Ubicación geográfica de la zona de estudio

denominado Acapetahua (Fig.3; CONAGUA 2009). Los niveles de los pozos que se alimentan de él son someros, con valores que van de los pocos centímetros a $10 \mathrm{~m}$ de profundidad (IMTA 2013).

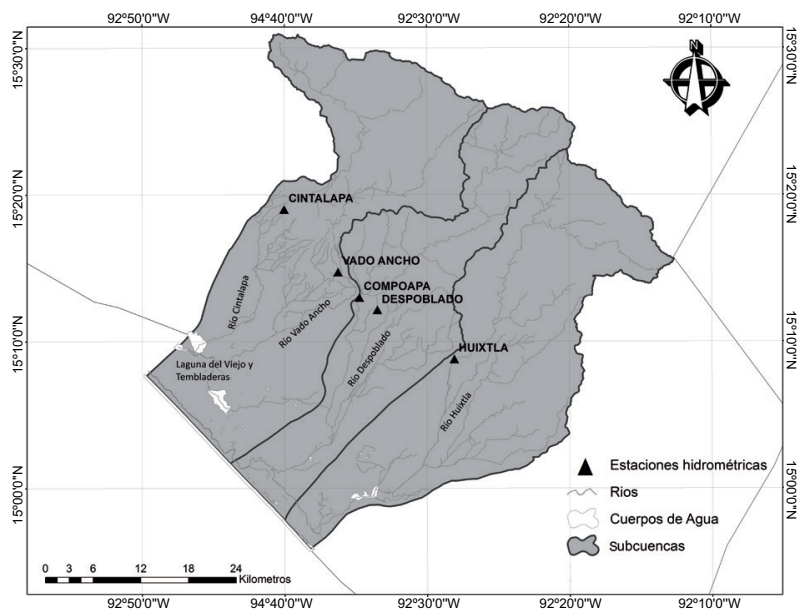

Fig. 2. Posición relativa de ríos principales y estaciones hidrométricas en la zona de estudio

\section{Modelo conceptual hidrogeológico}

Derivado de la información geológica y geofísica se deduce la presencia de un basamento hidrogeológico de rocas metamórficas de esquistos cristalinas, así como de rocas intrusivas graníticas de posible edad Precámbrica. Este basamento funciona como el límite inferior de los acuíferos de la zona (Centeno-

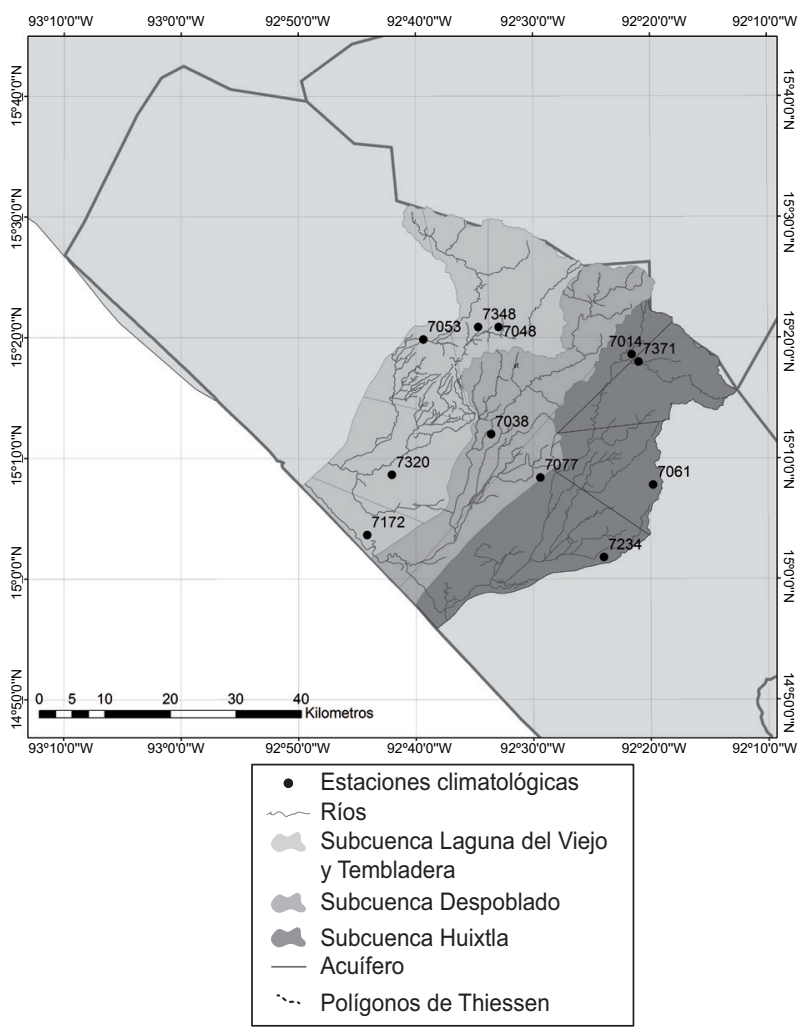

Fig. 3. Posición relativa estaciones climatológicas, sus respectivos polígonos de Thiessen y acuífero Acapetahua

García et al. 1993 y Castro-Mora 1999). La profundidad de este basamento es de pocos metros en las inmediaciones del pie montañoso hasta unos $120 \mathrm{~m}$ 


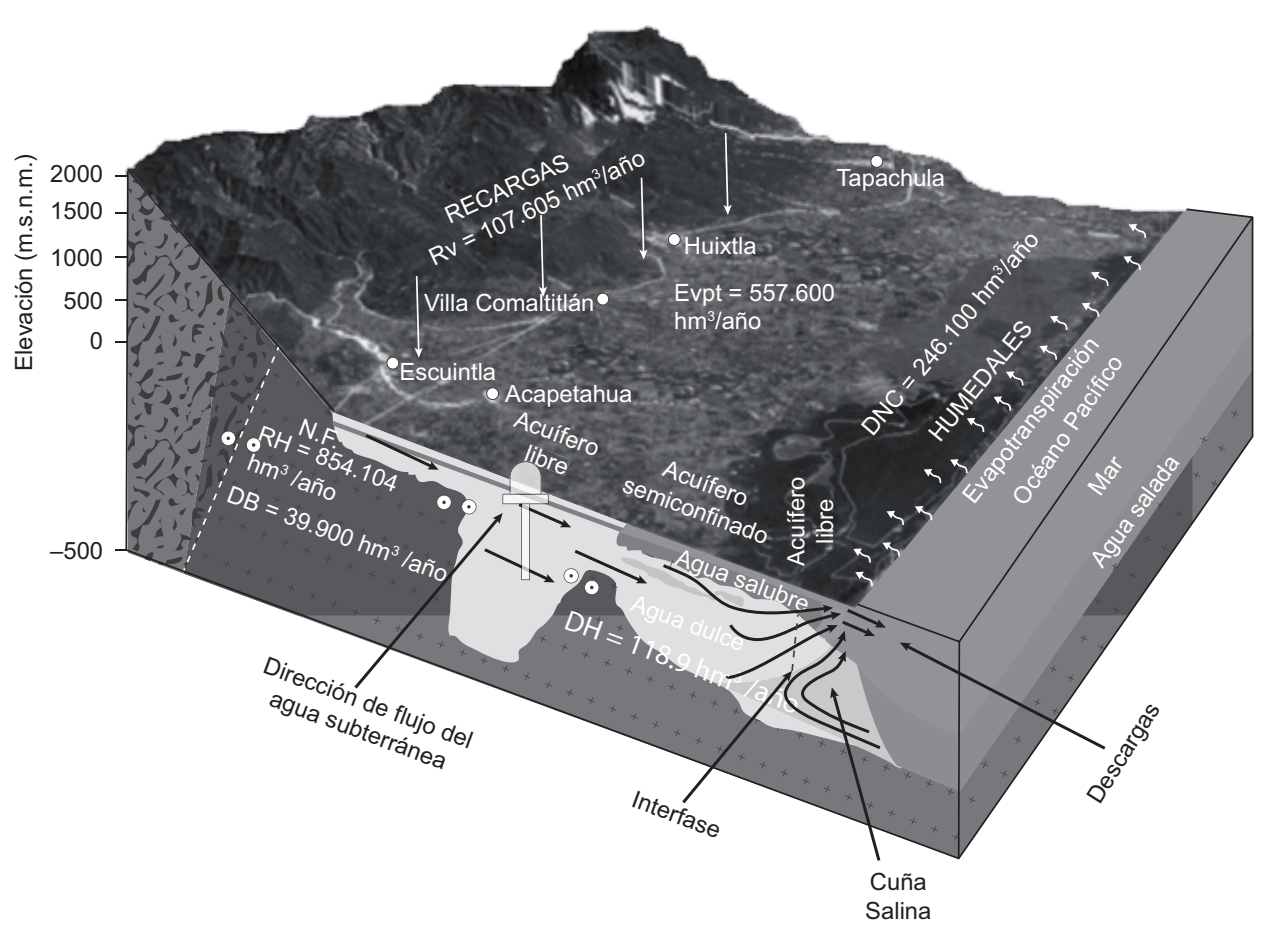

Fig. 4. Modelo conceptual de la zona de humedales de la costa de Chiapas, valores en $\mathrm{Mm}^{3} / \mathrm{año}$. $\mathrm{R}_{\mathrm{V}}=$ recarga vertical, $\mathrm{R}_{\mathrm{H}}=$ recarga horizontal, $\mathrm{D}_{\mathrm{NC}}=$ descargas naturales comprometidas, $\mathrm{D}_{\mathrm{B}}=$ bombeo, $\mathrm{Evpt}=$ evapotranspiración, $\mathrm{D}_{\mathrm{L}}=$ descargas laterales hacia el mar

cerca de la zona costera (Fig. 4). Sobreyaciendo a este basamento hidrogeológico se encuentran capas de sedimentos marinos depositados previamente al levantamiento de esta zona. Sin embargo, hasta la fecha no se han reportado evidencias de estos estratos. En la parte superior del relleno de la planicie costera se localizan intercalaciones de material granular que varían de gravas, arenas, limos y arcillas, los cuales corresponden a edades del Plioceno al Cuaternario.

El flujo del agua subterránea tiene una dirección este-oeste, recargándose en las partes altas montañosas y descargando hacia el mar. El agua fluye a través del material granular y alimenta de manera lateral a los humedales costeros de la RB La Encrucijada.

\section{Balance de aguas subterráneas}

El balance de aguas subterráneas (BAS) que a continuación se presenta corresponde esencialmente al acuífero de Acapetahua. Se fundamenta en el principio de conservación de la masa, cuya ecuación de continuidad establece que la diferencia entre los volúmenes de entradas (recarga) y salidas (descarga) de aguas subterráneas de un sistema, establecerá el posible cambio de volumen en el almacenamiento del sistema-acuífero para un determinado tiempo. Es decir: volúmenes de entrada - volúmenes de salida $=$ cambio del volumen almacenado

Como se ha señalado, las aguas subterráneas del acuífero Acapetahua se manifiestan de manera compleja a través de un sistema de medio granular (de comportamiento hidráulicamente libre y características heterogéneas) y anisótropas definidas. La profundidad, a la que se encuentra el nivel freático varía de pocos dm hasta unos $20 \mathrm{~m}$. La elevación del nivel freático es variable entre los 40 a $0 \mathrm{msnm}$, con un gradiente hidráulico de 0.002 en promedio en la zona de recarga. La dirección de flujo de las aguas subterráneas es del noreste (NE) al sureste (SE) en el área de estudio, transitando por el centro del valle hasta descargar lateralmente en la zona de humedales y al mar (IWA 2000). El espesor de saturación total de agua es en promedio de $600 \mathrm{~m}$. El basamento consiste de una superficie irregular, representada por roca masiva de origen ígneo/metamórfico, con escasa permeabilidad (prácticamente nula).

\section{Entradas}

Recarga lateral de aguas subterráneas

El fenómeno de la recarga lateral de las aguas subterráneas hacia el acuífero de Acapetahua, ocurre 
CUADRO I. ENTRADAS LATERALES DE VOLÚMENES DE AGUAS SUBTERRÁNEAS

\begin{tabular}{ccccccc}
\hline $\begin{array}{l}\text { Canal de } \\
\text { entrada }(\mathrm{e})\end{array}$ & $\begin{array}{c}\text { Longitud del } \\
\text { canal }(\mathrm{m})\end{array}$ & $\begin{array}{c}\text { Ancho del } \\
\text { canal }(\mathrm{m})\end{array}$ & Gradiente & $\begin{array}{c}\text { Conductividad } \\
\text { hidráulica }(\mathrm{m} / \mathrm{seg})\end{array}$ & $\begin{array}{c}\text { Caudal } \\
\left(\mathrm{m}^{3} / \mathrm{seg}\right)\end{array}$ & $\begin{array}{c}\text { Caudal } \\
\left(\mathrm{m}^{3} / \mathrm{año}\right)\end{array}$ \\
\hline e1 & 2941 & 180 & 0.0021 & 0.001 & 1.1254 & 35490067.3 \\
e2 & 7647 & 180 & 0.0028 & 0.001 & 3.9015 & 123038669 \\
e3 & 7058 & 180 & 0.0028 & 0.001 & 3.6010 & 113561780 \\
e4 & 7058 & 180 & 0.0042 & 0.001 & 5.4015 & 170342669 \\
e5 & 3529 & 180 & 0.0042 & 0.001 & 2.7008 & 85171334.7 \\
e6 & 2941 & 180 & 0.0021 & 0.001 & 1.1254 & 35490067.3 \\
e7 & 5882 & 180 & 0.0042 & 0.001 & 4.5015 & 141960269 \\
e8 & 2941 & 180 & 0.0028 & 0.001 & 1.5005 & 47320089.8 \\
e9 & 2941 & 180 & 0.0014 & 0.001 & 0.7500 & 23653340.4 \\
e10 & 3529 & 180 & 0.0021 & 0.001 & 1.3503 & 42585667.3 \\
e11 & 2941 & 180 & 0.0021 & 0.001 & 1.1253 & 35490067.3 \\
& & & & & Total & 854104022 \\
\hline
\end{tabular}

por continuidad hidráulica entre el material geológico de las zonas de montaña, que funciona como área de recarga (constituida principalmente por ígneas y metamórficas fracturadas) y el material sedimentario aluvial y lacustre que conforma el subsuelo de la planicie aluvial de este valle.

Para el cálculo de las entradas laterales, se utilizó la información potenciométrica registrada en el mes de diciembre del 2012. El resultado fue una red de flujo, donde las celdas de entradas laterales de aguas subterráneas ocurren de las estribaciones de las serranías del occidente de la planicie aluvial. Para cuantificar los volúmenes correspondientes, se aplicó la Ley de Darcy, que establece que el flujo de agua que pasa a través de una celda, es igual al producto de la conductividad hidráulica, espesor saturado de acuífero, ancho de la celda y gradiente hidráulico.

Las conductividades hidráulicas utilizadas, corresponden a las reportadas en la sección de pruebas de bombeo, además de las citadas en la literatura internacional especializada, para rocas semejantes a las emplazadas en el subsuelo, donde no se tienen evaluaciones de pruebas de bombeo. En el cuadro I se muestran los cálculos y resultados obtenidos, donde se observa que el volumen total anualizado de los flujos de aguas subterráneas que ingresan lateralmente al sistema, son del orden de los $854 \mathrm{Mm}^{3} /$ año. La red de flujo que respalda estos cálculos se presenta en la figura 5.

\section{Recarga vertical superficial}

Además de la recarga del agua subterránea que se genera en las zonas de montaña adyacentes y distantes, también existen los procesos de recarga vertical superficial dentro de la zona del BAS. Este fenómeno ocurre por la infiltración directa de las aguas de lluvia y a través del escurrimiento que generan. Sin embargo, para estimar los volúmenes de agua que

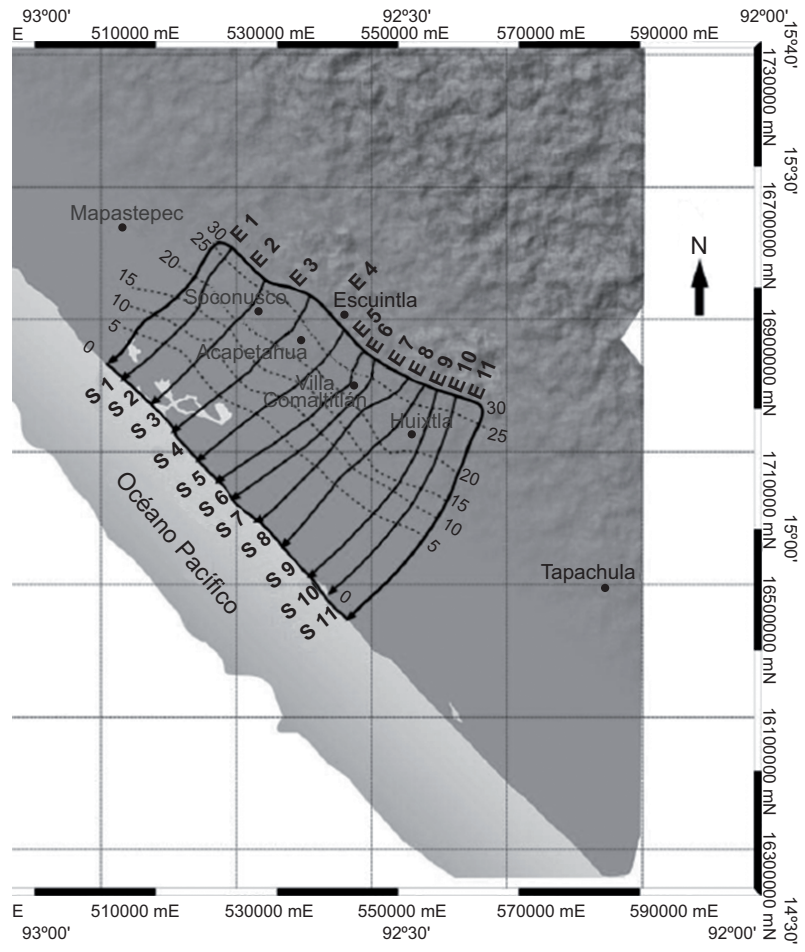

Fig. 5. Canales de entrada (E) y salida (S) de aguas subterráneas

efectivamente impactan en la cuota de renovación natural de las aguas subterráneas, habrá de considerar aquellos mecanismos que interactúan en este proceso, como es el efecto de la evapotranspiración. En este análisis se considera que el efecto de recarga vertical efectivo es nulo debido a la gran evapotranspiración.

\section{Salidas}

Para el cálculo del balance se toman en cuenta las salidas del acuífero, tanto naturales como artificiales. Entre los diferentes componentes del balance se 
CUADRO II. SALIDAS HORIZONTALES (DESCARGAS HACIA EL MAR) DEL ACUÍFERO DE ACAPETAHUA

\begin{tabular}{lccccrr}
\hline $\begin{array}{l}\text { Canal de } \\
\text { salida (s) }\end{array}$ & $\begin{array}{c}\text { Longitud } \\
\text { del canal }(\mathrm{m})\end{array}$ & $\begin{array}{c}\text { Ancho del } \\
\text { canal }(\mathrm{m})\end{array}$ & Gradiente & $\begin{array}{c}\text { Conductividad } \\
\text { hidráulica }(\mathrm{m} / \mathrm{s})\end{array}$ & $\begin{array}{r}\text { Caudal } \\
\left(\mathrm{m}^{3} / \mathrm{seg}\right)\end{array}$ & $\begin{array}{c}\text { Caudal } \\
\left(\mathrm{m}^{3} / \mathrm{año}\right)\end{array}$ \\
\hline s1 & 2941 & 205 & 0.0004 & 0.001 & 0.2697 & 8506268.83 \\
s2 & 5294 & 205 & 0.0004 & 0.001 & 0.2563 & 8081100.00 \\
s3 & 4705 & 205 & 0.0004 & 0.001 & 0.4393 & 13854062.00 \\
s4 & 5882 & 205 & 0.0003 & 0.001 & 0.3153 & 9944180.86 \\
s5 & 3529 & 205 & 0.0004 & 0.001 & 0.4658 & 14692227.90 \\
s6 & 3529 & 205 & 0.0003 & 0.001 & 0.2365 & 7458664.03 \\
s7 & 5294 & 205 & 0.0003 & 0.001 & 0.2562 & 8080527.56 \\
s8 & 5294 & 205 & 0.0004 & 0.001 & 0.4010 & 12648782.10 \\
s9 & 5882 & 205 & 0.0003 & 0.001 & 0.3690 & 11637223.60 \\
s10 & 3529 & 205 & 0.0003 & 0.001 & 0.3942 & 12431811.20 \\
s11 & 5882 & 205 & 0.0002 & 0.001 & 0.3416 & 10774189.40 \\
& & & & & Total & 118109038 \\
\hline
\end{tabular}

considera que las salidas naturales son a través de las descargas o flujo de agua subterránea que mantienen los humedales (descargas naturales comprometidas) y las descargas laterales hacia el mar. El componente artificial considerado son las descargas a través de los pozos y norias.

Descarga artificial (extracción de agua mediante pozos)

La extracción del agua subterránea se hace a través de pozos profundos para el abastecimiento público urbano y a través de norias que se distribuyen en prácticamente todas las casas de la zona de estudio. Se estimó que la extracción total en el acuífero es del orden de los $39.900 \mathrm{Mm}^{3} /$ año.

\section{Estimación de la descarga lateral}

La salida lateral estimada para el acuífero de Acapetahua fue aquella que descarga hacia el mar. Para ello se aplicó la ecuación de Darcy, estimándose una descarga hacia el mar del orden de $118.109 \mathrm{Mm}^{3} /$ año. Los cálculos se desglosan en el cuadro II.

Además, si se considera como un sistema en condiciones casi naturales (en equilibrio hidrológico), significa que los volúmenes de entrada serán iguales a los de salida. En este caso, se trata de egresos laterales subterráneos al sur, como lo evidencia la red de flujo del BAS.

\section{Evapotranspiración}

La evapotranspiración en la zona de estudio es muy alta al considerar los niveles tan someros del agua subterránea. Se estimó que el volumen de evapotranspiración es del orden de $557.6 \mathrm{Mm}^{3} /$ año (CONAGUA 2009)

\section{Variación en el almacenamiento de las aguas subterráneas}

Un cambio de volumen en el almacenamiento de las aguas subterráneas existirá cuando en un cierto lapso de tiempo se presenten modificaciones en los volúmenes de entrada o salida de un sistema. El acuífero de Acapetahua, se considera un sistema en equilibrio ya que los volúmenes extraídos artificialmente son mínimos en comparación con los de entrada lateral o vertical estimados. Las variaciones que se aprecian corresponden a los ciclos de lluvia y estiaje, no se identifican conos de abatimiento. Se analizaron las configuraciones del nivel estático para los años 1981 y 2012 (Fig. 6), sin embargo no se aprecia un cambio significativo en las curvas. Por tanto, las variaciones del almacenamiento de las aguas subterráneas son mínimas.

\section{Cálculo del balance hidrogeológico}

Como ya se mencionó, el BAS se fundamenta por la ecuación de continuidad, que establece que la diferencia entre las magnitudes de entradas (recargas) y salidas (descargas) de un sistema-acuífero será el cambio de almacenamiento. Dicha ecuación, puede plantearse a través de la siguiente relación entre volúmenes, quedando como incógnita por identificar la recarga vertical del sistema-acuífero:

$\left(R_{L}+R_{V}\right)-\left(D_{L}+D_{B}+D_{N}+E_{v p t}\right)=\Delta V=0 \ldots$

$R_{V}=\left(D_{L}+D_{B}+D_{N}+E_{v p t}\right)-R_{L}$

$R_{V}=(118.109+39.9+246.1+557.6)-854.104$

$R_{V}=961.709-854.104$

$R_{V}=107.605 \mathrm{Mm}^{3} / \mathrm{a}$ 


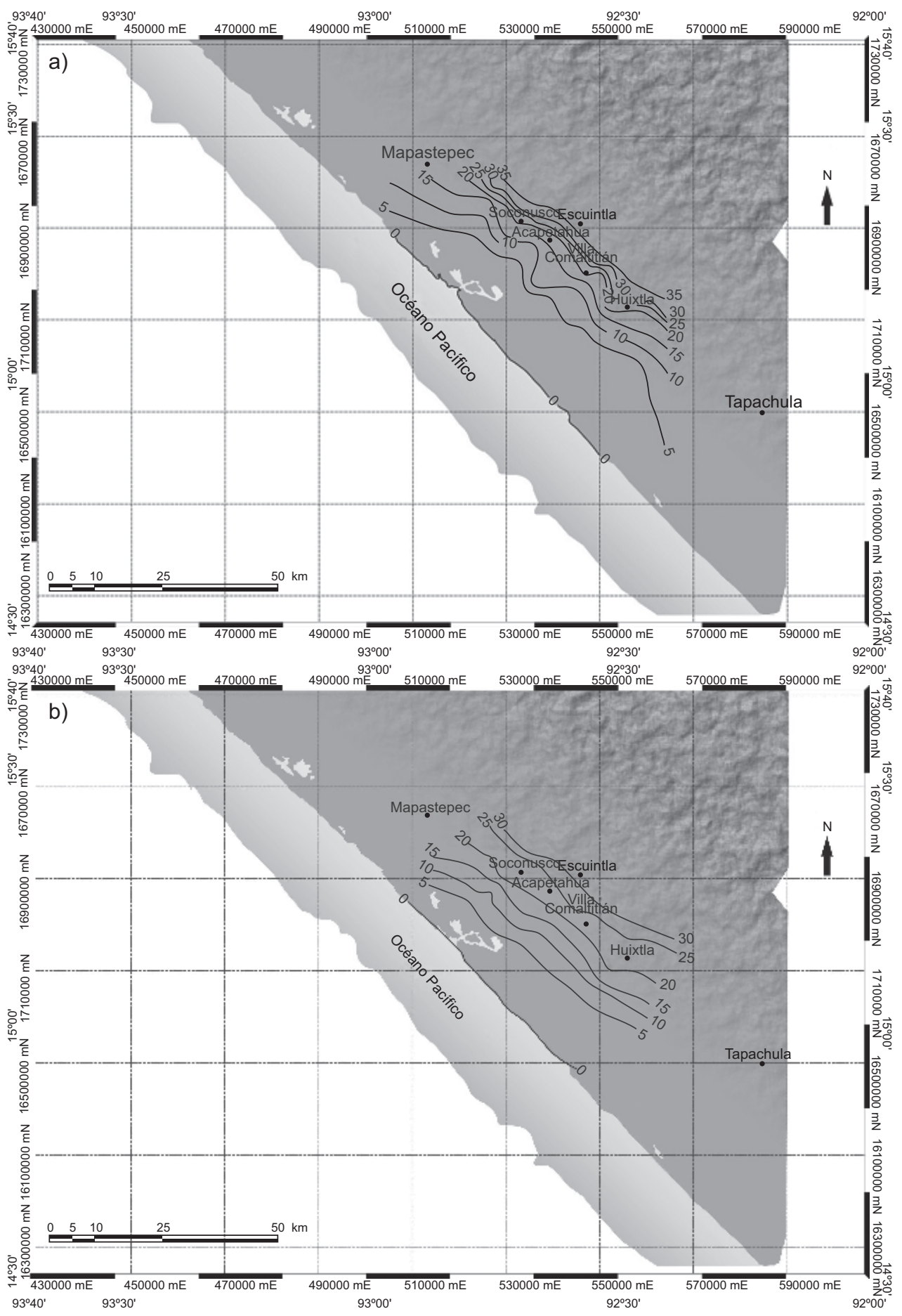

Fig. 6. Elevación de nivel estático (msnm): a) 1981, b) 2012

Dónde:

$R_{L}=$ recarga lateral al sistema-acuífero

$R_{V}=$ recarga vertical superficial

$D_{L}=$ descarga lateral hacia un sistema contiguo

$D_{B}=$ descarga del sistema-acuífero por bombeo
$\Delta V=$ cambio en el volumen del almacenamiento del sistema-acuífero

Como se puede advertir, existe una recarga vertical anual del sistema-acuífero del orden de $107.605 \mathrm{Mm}^{3}$, mientras que la recarga total media anual (que es la 
suma de la recarga horizontal subterránea 854.104 $\mathrm{Mm}^{3}$ y la recarga vertical $107.605 \mathrm{Mm}^{3}$ ) es de $961.709 \mathrm{Mm}^{3}$.

Para el caso específico de los humedales de Acapetahua se propone que las descargas naturales comprometidas incluyan tanto el volumen de alimentación a manantiales/humedales como a las descargas hacia el mar para que se mantenga el equilibrio entre el agua dulce y el agua salada. Así, el volumen de descargas comprometidas serían del orden de $364.209 \mathrm{Mm}^{3} /$ año $\left(D_{N}=246.1 \mathrm{Mm}^{3} /\right.$ año más $D_{L}=118.109 \mathrm{Mm}^{3} /$ año).

\section{Cálculo de disponibilidad}

Para el cálculo de la disponibilidad de las aguas subterráneas en el sistema-acuífero de Acapetahua, se aplica el procedimiento indicado en la Norma Oficial Mexicana NOM-011-CNA-2015 (CONAGUA 2015), que establece las especificaciones y el método para determinar la disponibilidad media anual de las aguas nacionales, que en la fracción relativa a las aguas subterráneas establece que:

$D_{M A}=R_{T M A}-D_{N C}-V_{R E P D A}$

Dónde:

$D_{M A}=$ disponibilidad media anual de agua subterránea en una unidad hidrogeológica ( $\left.\mathrm{Mm}^{3} / \mathrm{año}\right)$

$R_{T M A}=$ recarga total media anual $\left(\mathrm{Mm}^{3} / \mathrm{año}\right)=$ $961.709 \mathrm{Mm}^{3} /$ año

$D_{N C}=$ descarga natural comprometida $\left(\mathrm{Mm}^{3} / \mathrm{a}\right)=$ $364.209 \mathrm{Mm}^{3}$ /año

$V_{R E P D A}=$ volumen anual de agua subterránea concesionado e inscrito en el registro público de derechos de agua $($ REPDA $)\left(\mathrm{Mm}^{3} / \mathrm{año}\right)=63.583 \mathrm{Mm}^{3} /$ año

Sustituyendo en la ecuación anterior:

$D_{M A}=961.709-364.209-63.583$

Es decir:

$D_{M A}=533.917 \mathrm{Mm}^{3} /$ año

El valor obtenido corresponde al volumen que potencialmente la CONAGUA podría concesionar. Cabe señalar que dicho volumen protege al acuífero y al volumen que sustenta a los humedales (los cuales de acuerdo con la ley de aguas nacionales, se denominan descargas naturales comprometidas).

Por tanto, existe una disponibilidad en el acuífero de Acapetahua de $533.917 \mathrm{Mm}^{3} /$ año.

\section{Balance de aguas superficiales Estimación del escurrimiento a través del método del número de curva}

En la figura 2 se observa la posición relativa de las estaciones hidrométricas que registran el escurrimiento en los cuatro cauces principales de la zona de interés. Asimismo es evidente que una parte de la red por la que escurre una fracción importante de la precipitación no concurre o transita por los cuatro puntos donde se encuentran tales estaciones hidrométricas.

Lo anterior implica un registro parcial hidrométrico del escurrimiento para la zona de estudio, por ello se estimó el escurrimiento en toda la zona a través de un método indirecto al utilizar el registro de precipitación de las estaciones climatológicas que aparecen en la figura 3. Dicho método es el número de curva (CN, por sus siglas en inglés) del Servicio de Conservación de los Recursos Naturales (Natural Resources Conservation Service, antes Soil Conservation Service (SCS); Hawkins et al. 2009).

Este es un método empírico para el cálculo de la transformación de lluvia a escurrimiento, que surgió de la observación del fenómeno hidrológico en distintos tipos de suelo y para distintas condiciones de humedad antecedente. Con los dos últimos parámetros se observaron curvas al representarse en forma gráfica la profundidad de precipitación $(\mathrm{P})$ y la profundidad de exceso de precipitación o escurrimiento directo (Pe). Para estandarizar estas curvas, se determinó un número adimensional de $\mathrm{CN}$, tal que $0<=\mathrm{CN}<=$ 100. Se definió que para superficies impermeables y agua $\mathrm{CN}=100$, para superficies naturales $\mathrm{CN}<100$ y para superficies sin escurrimiento $\mathrm{CN}=0$.

Los $\mathrm{CN}$ se definieron según las condiciones antecedentes de humedad: condición normal (condición II), condición seca (condición I) y condición húmeda (condición III), en cada caso existe una expresión algebraica que permite determinar dicho parámetro.

Este modelo asume la hipótesis de que el escurrimiento acumulado en una porción de la cuenca es proporcional a la infiltración acumulada, como la precipitación bruta acumulada una vez descontada la fracción necesaria para que se produzca el encharcamiento:

$\frac{Q}{F}=\frac{P-P_{0}}{S}$

Donde:

$\mathrm{S}=$ máxima infiltración potencial, $\mathrm{F}=$ infiltración real después del encharcamiento,

$\mathrm{P}=$ precipitación,$\quad \mathrm{Q}=$ escurrimiento superficial y $\mathrm{P}_{0}=$ umbral de escurrimiento, es decir, la cantidad de precipitación necesaria para que el suelo se encharque y el escurrimiento superficial aparezca.

Los estudios empíricos realizados por el SCS permitieron relacionar la máxima infiltración potencial 
con un parámetro de referencia denominado número de curva $(\mathrm{CN})$, cuyos valores están tabulados entre 0 y 100 .

$S=\frac{1000}{C N}-10$

Los resultados de escurrimiento diario fueron acumulados con una frecuencia mensual para cada subcuenca. Posteriormente estos valores se compararon con el registro mensual de las estaciones hidrométricas que se tienen por subcuenca.

Como se esperaba, los valores del cálculo de escurrimiento a través de la precipitación son mayores a los registrados en las estaciones hidrométricas. Asimismo existen registros de escurrimiento en las estaciones hidrométricas durante el periodo que no ocurre lluvia y cuya estimación a través del método antes descrito tiene valor cero.

Estos periodos definidos a partir de la comparación del registro hidrométrico y del registro estimado, nos definen temporalmente los periodos en que la fuente subterránea es la principal fuente de abastecimiento para la RB La Encrucijada y los volúmenes así definidos nos indican el valor mínimo que aporta la mencionada fuente hacia aguas abajo.

\section{RESULTADOS Y DISCUSIÓN}

El cálculo del escurrimiento se realizó por subcuenca con una frecuencia temporal diaria. Se empleó el registro de precipitación total disponible de las estaciones climatológicas indicadas en la figura 3. Además, se emplearon los polígonos de Thiessen que aparecen en la citada figura para delimitar el área de influencia de cada estación climatológica con su registro de precipitación.

El método considera la humedad antecedente del suelo durante los últimos cinco días, con dicha condición se selecciona el CN a utilizar de acuerdo con tres posibles condiciones de suelo mencionadas previamente en la sección de materiales y métodos: condición I, condición II ó condición III. Una vez con los resultados anteriores se realizó el cálculo del escurrimiento.

Sumado a lo anterior es imprescindible y de manera previa establecer el $\mathrm{CN}$ de acuerdo con su condición física. Es decir, atendiendo al tipo de suelo y su cobertura, por esta razón se hizo una clasificación del mismo con la información disponible para el área de estudio, ajustando la información a las clases habituales que se utilizan en el cálculo del escurrimiento por este método. La descripción resumida por subcuenca y por polígono de Thiessen se describe a continuación:

\section{Subcuenca del río Despoblado}

Para esta subcuenca se emplearon los registros de dos estaciones climatológicas, sus respectivas áreas de influencia, el número de clases de cobertura (tipos de suelo) existentes dentro de dicha área de influencia y el $\mathrm{CN}$ ponderada, calculado a partir del número de clases (tipos de cobertura) y el área de las mismas, así como el periodo de registro disponible por estación (Cuadro III).

\section{Subcuenca Laguna del Viejo y Tembladeras}

Para esta subcuenca se emplearon los registros de cinco estaciones climatológicas, sus respectivas áreas de influencia, el número de clases de cobertura (tipos de suelo) existentes dentro de dicha área de influencia y el CN ponderada, calculado a partir del número de clases (tipos de cobertura) y el área de las mismas, así como el periodo de registro disponible por estación (Cuadro IV).

\section{Subcuenca del río Huixtla}

Para esta subcuenca se emplearon los registros de cinco estaciones climatológicas, sus respectivas áreas de influencia, el número de clases de cobertura (tipos de suelo) existentes dentro de dicha área de influencia y el CN ponderada, calculado a partir del número de clases (tipos de cobertura) y el área de las mismas, así como el periodo de registro disponible por estación (Cuadro V).

CUADRO III. INFORMACIÓN BÁSICA PARA EL CÁLCULO DEL ESCURRIMIENTO EN LA SUBCUENCA DEL RÍO DESPOBLADO

\begin{tabular}{lcccc}
\hline $\begin{array}{l}\text { Estación } \\
\text { climatológica }\end{array}$ & $\begin{array}{c}\text { Área de } \\
\text { influencia }\left(\mathrm{km}^{2}\right)\end{array}$ & $\begin{array}{c}\text { Número de clases } \\
\text { de cobertura }\end{array}$ & $\begin{array}{c}\text { Número de curva } \\
\text { ponderada }\end{array}$ & Registro disponible \\
\hline 7077 & 139.6 & 16 & 78.3 & ene 1964- dic 2011 \\
7038 & 464.7 & 21 & 77.5 & oct 1964- dic 2000 \\
\hline
\end{tabular}


CUADRO IV. INFORMACIÓN BÁSICA PARA EL CÁLCULO DEL ESCURRIMIENTO EN LA SUBCUENCA DE LAGUNA DEL VIEJO Y TEMBLADERAS

\begin{tabular}{lcccc}
\hline $\begin{array}{l}\text { Estación } \\
\text { climatológica }\end{array}$ & $\begin{array}{c}\text { Área de } \\
\text { influencia }\left(\mathrm{km}^{2}\right)\end{array}$ & $\begin{array}{c}\text { Número de } \\
\text { clases de cobertura }\end{array}$ & $\begin{array}{c}\text { Número de } \\
\text { curva ponderada }\end{array}$ & Registro disponible \\
\hline 7172 & 88.9 & 10 & 90.7 & oct 1965-sep 2009 \\
7348 & 144.6 & 13 & 79.5 & jun 1981-sep 2009 \\
7053 & 163.4 & 13 & 77.3 & mar 1964-nov 2007 \\
7048 & 216.1 & 10 & 80.4 & feb 1966-sep 2008 \\
7320 & 278.3 & 14 & 79.0 & abr 1978-nov 2009 \\
\hline
\end{tabular}

CUADRO V. INFORMACIÓN BÁSICA PARA EL CÁLCULO DEL ESCURRIMIENTO EN LA SUBCUENCA DEL RÍO HUIXTLA

\begin{tabular}{lcccc}
\hline $\begin{array}{l}\text { Estación } \\
\text { Climatológica }\end{array}$ & $\begin{array}{c}\text { Área de } \\
\text { influencia }\left(\mathrm{km}^{2}\right)\end{array}$ & $\begin{array}{c}\text { Número de clases } \\
\text { de cobertura }\end{array}$ & $\begin{array}{c}\text { Número de curva } \\
\text { ponderada }\end{array}$ & Registro disponible \\
\hline 7014 & 93.3 & 14 & 78.0 & may 1964-sep 2001 \\
7061 & 178.3 & 11 & 81.7 & abr 1964-oct 2010 \\
7371 & 205.2 & 12 & 77.4 & ene 1980-ene 2000 \\
7324 & 351.5 & 14 & 77.5 & jun 1977-nov 2001 \\
\hline
\end{tabular}

En cada subcuenca el cálculo del escurrimiento se realizó con una frecuencia diaria, el inconveniente de ésto reside en que el registro continuo de precipitación en cada una las distintas estaciones climatológicas no coincide con las restantes de la subcuenca. Es decir, existen periodos faltantes de información en cada registro por estación y estos periodos no son los mismos, situación habitual en los registros de nuestro país. Por tanto, en cada subcuenca se buscó un periodo temporal diario continuo (sin faltantes) que fuera común para todo el grupo de estaciones dentro de la subcuenca. Dicho periodo fue el utilizado para calcular el escurrimiento diario a través de la precipitación. Posteriormente se realizó la suma acumulada con una frecuencia mensual de ese escurrimiento calculado, considerando en dicha suma todos los escurrimientos calculados por los registros de precipitación dentro de la subcuenca expresado en unidades de volumen. Esto último obedece a que se dispone del registro del escurrimiento acumulado mensual en la subcuenca (registro hidrométrico), con lo cual fue factible realizar una comparación mes a mes del volumen de escurrimiento calculado y el volumen de escurrimiento medido.

A través de la diferencia del volumen calculado menos el volumen medido, es posible establecer cuánto volumen proviene de la fuente subterránea bajo la siguiente consideración: si la diferencia entre ambos valores es de signo negativo implica que el volumen medido es superior, lo que indica que ese volumen (diferencia) es de origen subterráneo, esto se verificó con el registro de precipitación para esos valores mensuales. Se encontró que en varios días o incluso en todo el mes, no hay registro de precipitación.

\section{Con lo reseñado hasta aquí tenemos que:}

1. Los periodos durante los cuales el escurrimiento calculado es cero, dada la nula o escasa precipitación, pero el registro hidrométrico tiene valores diferentes de cero, implica que ese volumen corresponde al gasto base en los cauces y su origen es el agua subterránea.

2. El cuadro VI muestra por subcuenca los volúmenes mínimo, máximo y promedio, tanto de agua superficial como de agua subterránea en la zona de estudio. Se observa que el volumen promedio cuya fuente es subterránea para la zona de estudio es de $295.852 \mathrm{hm}^{3}$ por año $\left(17.934 \mathrm{hm}^{3}+132.978 \mathrm{hm}^{3}+\right.$ $144.94 \mathrm{hm}^{3}$ ). El valor estimado como descarga natural en el balance de agua subterránea es de $246.1 \mathrm{hm}^{3}$ por año, entre los dos últimos valores citados hay una diferencia de $20 \%$.

5. Los resultados anteriores se obtuvieron a pesar de que no hubo una coincidencia en los periodos temporales de los registros de precipitación para cada grupo de estaciones, en las tres subcuencas. 
CUADRO VI. ESTADÍSTICOS DE LOS VOLÚMENES PROMEDIO DE AGUAS SUPERFICIALES Y SUBTERRÁNEAS POR SUBCUENCA

\begin{tabular}{|c|c|c|c|c|c|c|c|c|c|}
\hline \multirow[t]{2}{*}{ Estadístico } & \multicolumn{3}{|c|}{ Subcuenca del río Despoblado } & \multicolumn{3}{|c|}{ Subcuenca Laguna del Viejo y Tembladeras } & \multicolumn{3}{|c|}{ Subcuenca del río Huixtla } \\
\hline & $\begin{array}{l}\text { Vol. Subt. } \\
\left(\mathrm{hm}^{3}\right)\end{array}$ & $\begin{array}{l}\text { Vol. Sup. } \\
\left(\mathrm{hm}^{3}\right)\end{array}$ & $\begin{array}{l}\text { Vol. Tot. } \\
\left(\mathrm{hm}^{3}\right)\end{array}$ & $\begin{array}{l}\text { Vol. Subt. } \\
\left(\mathrm{hm}^{3}\right)\end{array}$ & $\begin{array}{l}\text { Vol. Sup. } \\
\left(\mathrm{hm}^{3}\right)\end{array}$ & $\begin{array}{l}\text { Vol. Tot. } \\
\left(\mathrm{hm}^{3}\right)\end{array}$ & $\begin{array}{l}\text { Vol. Subt. } \\
\left(\mathrm{hm}^{3}\right)\end{array}$ & $\begin{array}{l}\text { Vol. Sup. } \\
\left(\mathrm{hm}^{3}\right)\end{array}$ & $\begin{array}{c}\text { Vol. Tot. } \\
\left(\mathrm{hm}^{3}\right)\end{array}$ \\
\hline Mínimo & 8.266 & 491.289 & 507.274 & 19.208 & 8.176 & 59.154 & 99.083 & 34.993 & 99.083 \\
\hline Máximo & 40.061 & 827.438 & 848.154 & 310.024 & 517.224 & 675.704 & 193.847 & 110.641 & 304.489 \\
\hline Promedio & 17.934 & 603.789 & 621.724 & 132.978 & 262.136 & 395.114 & 144.940 & 60.432 & 190.263 \\
\hline Desviación & 6.976 & 109.188 & 110.217 & 95.430 & 132.636 & 172.689 & 43.247 & 43.484 & 87.000 \\
\hline
\end{tabular}

Vol. Subt. = volumen subterráneo, Vol. Sup. = volumen superficial, Vol. Tot. = volumen total

\section{CONCLUSIONES}

- La ubicación de las estaciones hidrométricas a pie de la carretera Arriaga-Tapachula (aproximadamente en la parte media de la zona de estudio), limita tener un registro directo de la mayor parte del agua que escurre hacia la zona baja y por ende al humedal.

- En la parte superficial, es evidente que existe suficiente volumen de agua para la permanencia del humedal bajo sus condiciones actuales. Sin embargo, existen problemas en la calidad del agua de depósito, arrastre y transporte de sedimentos, además de que estos tres últimos procesos se han incrementado por la construcción de pedraplenes en los taludes de las márgenes de los cauces principales, mismos que modifican la dinámica de los ríos. Adicionalmente, se permite la extracción del material de los cauces (grava y arena, la proliferación de casas de materiales en la zona es evidente), lo que augura más modificaciones al comportamiento de los ríos. Es probable que se generen problemas en el mediano plazo de diferente índole -no sólo hidrológica- a causa de esta alteración que no ha sido valorada. La parte lagunar en los últimos años ha experimentado un incremento visible del depósito de materiales, con la pérdida de profundidad en la misma.

-El cálculo de los componentes del balance de aguas subterráneas indica que el acuífero tiene una disponibilidad de agua subterránea del orden de $533.9 \mathrm{Mm}^{3} /$ año, es decir un volumen concesionable de acuerdo con la ley de aguas nacionales. El rendimiento seguro es aún mayor ya que corresponde al volumen de recarga menos las descargas naturales comprometidas y es del orden de $597.5 \mathrm{Mm}^{3} /$ año. Sin embargo, por la naturaleza costera del acuífero se deberá considerar que cualquier desarrollo no planificado podría modificar al sistema.

-La aproximación (diferencia del $20 \%$ ) entre los dos valores del volumen que en promedio terminan en la zona del humedal, calculados de diferente manera, dan la certidumbre de que los valores son aceptables. Lo anterior debido a que su obtención fue a partir del escurrimiento superficial, por lo que está respaldado de manera implícita por la utilización de los registros hidrométricos.

Como se mencionó en la parte final del apartado de resultados y discusión, el valor referido proviene de un análisis a nivel diario en primera instancia y luego de un acumulado mensual, con lo cual, en dicho valor se incluyen el registro de las variaciones a lo largo de diferentes años del volumen en la zona de estudio.

\section{AGRADECIMIENTOS}

Se agradece la participación de la Ingeniera María Eugenia Maya Magaña, por la elaboración de las figuras 1, 2 y 3 del presente escrito.

\section{REFERENCIAS}

Castro-Mora J. (1999). Monografía geológico-minera del estado de Chiapas. Consejo de Recursos Minerales, Ciudad de México, México, 180 pp.

Centeno-García E., Ruíz J., Coney P. J., Patchett P. J. y Ortega-Gutiérrez F. (1993). Guerrero terrene of Mexico: Its role in the southern cordillera from new geochemical data. Geology (21), 419-422.

CONAGUA (2009). Actualización de la disponibilidad media anual de agua subterránea del acuífero de Acapetahua (0709), Estado de Chiapas. Comisión Nacional del Agua. Diario Oficial de la Federación. 28 de agosto de 2009.

CONAGUA (2011). Identificación de reservas potenciales de agua para el medio ambiente en México. Informe. Comisión Nacional del Agua. Ciudad de México, México, 86 pp. 
CONAGUA (2014). Reservas potenciales de agua para el medio ambiente. Mapas interactivos. Comisión Nacional del Agua [en línea]. http://siga.cna.gob.mx/ sections/antecedentes.html 04/03/2015.

CONAGUA (2015). Norma Oficial Mexicana NOM-011CNA-2015. Conservación del recurso agua que establece las especificaciones y el método para determinar la disponibilidad media anual de las aguas nacionales. Comisión Nacional del Agua. Diario Oficial de la Federación. 27 de marzo de 2015.

Hawkins R. H., Ward T. J., Woodward D. E. y Van Mullen J. A. (2009). Curve number hydrology: State of the practice. American Society of Civil Engineers, Reston, EUA, 106 pp.

IMTA (2010). Extractor rápido de información climatológica (ERIC III). Instituto Mexicano de Tecnología del Agua. Base de datos, Jiutepec, Morelos, México, CD-ROM

IMTA (2013). Inventario y programa de manejo integral para la conservación de los humedales del Soconusco a través de su delimitación, caracterización ecológica, hidrológica, social y grado de riesgo. Informe. Instituto Mexicano de Tecnología del Agua. Jiutepec, Morelos, México, $512 \mathrm{pp}$.

INEGI (2011). Simulador de flujos de agua de cuencas hidrográficas (SIATL). Instituto Nacional de
Estadística y Geográfía. Mapas Interactivos [en línea]. http://antares.inegi.org.mx/analisis/red_hidro/ SIATL/\#app $=f 4 c 9 \&$ e312-selectedIndex $=0 \& 7 \mathrm{~b} 02$ selectedIndex $=0$ 14/01/2014.

IWA (2000). Specialist group on use of macrophytes in water pollution control. Constructed wetlands for pollution control: Process, performance, design, and operation. Reporte Técnico 8. The International Water Association. Londres, Reino Unido, 164 pp.

SE (2012). Norma Mexicana NMX-AA-159-SCFI-2012. Norma Mexicana que establece el procedimiento para la determinación del caudal ecológico en cuencas hidrológicas. Secretaría de Economía. Diario Oficial de la Federación. 20 de septiembre de 2012.

Salinas R. S. A. (2015). Programa nacional de reservas de agua: resultados alcanzados durante la primera fase (2012-2014). Memorias. V Congreso Mexicano de Ecología. San Luis Potosí, San Luis Potosí, México, 19 al 24 de abril 2015, CD-ROM.

SEMARNAT, CONAGUA, IMTA (2008). Banco nacional de datos de aguas superficiales sistema de información de aguas superficiales. Base de datos. Secretaría del Medio Ambiente y Recursos Naturales, Comisión Nacional del Agua, Instituto Mexicano de Tecnología del Agua. Jiutepec, Morelos, México, CD-ROM 